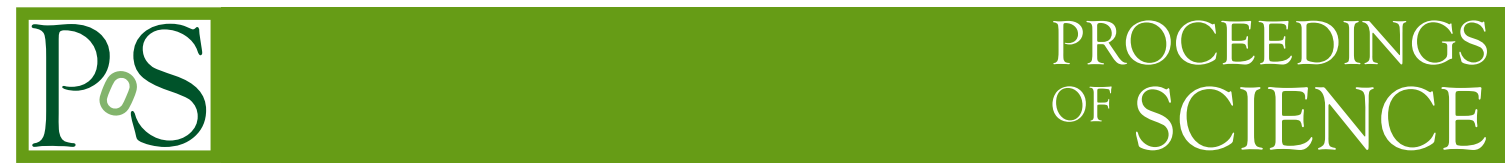

\title{
Recent BABAR studies of bottomonium states
}

\author{
Elisa Guido*i \\ INFN - Sezione di Genova, Via Dodecaneso 33 I-16146 Genova (Italy) \\ E-mail: elisa.guido@ge.infn.it
}

\begin{abstract}
We present the search for the $h_{b}(1 P)$ state of bottomonium in the transitions $\Upsilon(3 S) \rightarrow \pi^{0} h_{b}$ and $\Upsilon(3 S) \rightarrow \pi^{+} \pi^{-} h_{b}$ using a data sample of 122 million $\Upsilon(3 S)$ resonances. Dipion inclusive transitions between bottomonia are also studied with high precision. Moreover we present a study of the radiative transitions from decays of the $\Upsilon(2 S)$ and $\Upsilon(3 S)$ resonances using photons that have converted into an $e^{+} e^{-}$pair, obtaining precise measurements of the branching fractions of the transitions $\chi_{b 1,2}(1 P, 2 P) \rightarrow \gamma \Upsilon(1 S)$ and $\chi_{b 1,2}(2 P) \rightarrow \gamma \Upsilon(2 S)$, and searching for radiative decay to the $\eta_{b}(1 S)$ and $\eta_{b}(2 S)$ states.
\end{abstract}

The 2011 Europhysics Conference on High Energy Physics, EPS-HEP 2011,

July 21-27, 2011

Grenoble, Rhône-Alpes, France

*Speaker.

${ }^{\dagger}$ On behalf of the BABAR Collaboration. 


\section{Introduction}

The BABAR experiment - which is described in detail elsewhere $[1,2]$ - has recently obtained several important results in bottomonium physics, mainly based on the large datasets collected at energies in the $e^{+} e^{-}$center-of-mass (CM) frame equal to the masses of the $\Upsilon(3 S)$ and $\Upsilon(2 S)$ resonances. The integrated luminosities of the samples analyzed are of $28.0 \mathrm{fb}^{-1}$ at the $\Upsilon(3 S)$, and $13.6 \mathrm{fb}^{-1}$ at the $\Upsilon(2 S)$, respectively. Here we show the results obtained in the search for the $h_{b}(1 P)$ state, with the evidence of the decays $\Upsilon(3 S) \rightarrow \pi^{0} h_{b}(1 P)$ [3], and the search for the same state in the decays $\Upsilon(3 S) \rightarrow \pi^{+} \pi^{-} h_{b}(1 P)$ [4]. We also present a study of radiative bottomonium transitions using converted photons [5].

\section{Search for $h_{b}(1 P)$ in $\Upsilon(3 S)$ decays}

After the thirty-year-long search for the $\eta_{b}(1 S)$ bottomonium state [6], the interest in the bottomonium spectroscopy has been revitalized, and focused on the search for the still missing resonances. Here we present the recent results obtained by $B A B A R$ in the search for the $h_{b}(1 P)$ state, using the sample of $\Upsilon(3 S)$ mesons.

Measuring mass and properties of the $h_{b}(1 P)$ is fundamental in order to understand the hyperfine mass splitting for $P$-wave states. The expected mass of the $h_{b}(1 P)$ is the spin-weighted center-of-gravity of the $\chi_{b J}(1 P)$ system, i.e. about $9900 \mathrm{MeV} / \mathrm{c}^{2}$. The predicted decay mechanism from the $\Upsilon(3 S)$ are $\Upsilon(3 S) \rightarrow \pi^{0} h_{b}(1 P)$ with a rate of $10^{-3}$ [7], and $\Upsilon(3 S) \rightarrow \pi^{+} \pi^{-} h_{b}(1 P)$, with a rate ranging from $10^{-2}$ to $10^{-3}$ [8]. The $h_{b}(1 P)$ is expected [9] to decay mainly through three gluons $(57 \%)$, and radiatively to the $\eta_{b}(1 S)(41 \%)$.

\subsection{Evidence for $\Upsilon(3 S) \rightarrow \pi^{0} h_{b}(1 P)$ decays}

We analyze the events $\Upsilon(3 S) \rightarrow \pi^{0} h_{b}(1 P)$ with $h_{b}(1 P) \rightarrow \gamma \eta_{b}(1 S)$, where the photon energy range is well defined by the precision measurement of the $\eta_{b}(1 S)$ mass [6]. In order to extract the signal, we consider the mass distribution of the system recoiling against the $\pi^{0}$ candidate, defined as: $m_{\text {recoil }}\left(\pi^{0}\right)=\sqrt{\left(\sqrt{s}-E^{*}\left(\pi^{0}\right)\right)^{2}-p^{*}\left(\pi^{0}\right)^{2}}$, where $\sqrt{s}$ indicates the CM energy, and $E^{*}\left(\pi^{0}\right)$ and $p^{*}\left(\pi^{0}\right)$ the energy and the momentum of the $\pi^{0}$ in the CM frame. We fit the two-photon invariant mass in intervals of $m_{\text {recoil }}\left(\pi^{0}\right)$ in order to determine the number of $\pi^{0}$ in each interval. The $m_{\text {recoil }}\left(\pi^{0}\right)$ distribution on data is fitted with a smooth background component plus a signal component. After the background subtraction, the distribution is shown in Fig. 1 (a); a peaking structure attributed to the $h_{b}(1 P)$ signal is present, with a statistical significance of $3.8 \sigma$. After considering the systematic uncertainties, mainly due to the signal and background descriptions, the significance reduces to $3.1 \sigma$, leading to an evidence for the $h_{b}(1 P)$ signal. We measure a mass equal to: $m\left(h_{b}(1 P)\right)=9902 \pm 4$ (stat.) \pm 2 (syst.) $\mathrm{MeV} / \mathrm{c}^{2}$, and a product of branching fractions: $\mathscr{B}\left(\Upsilon(3 S) \rightarrow \pi^{0} h_{b}(1 P)\right) \times \mathscr{B}\left(h_{b}(1 P) \rightarrow \gamma \eta_{b}(1 S)\right)=(4.3 \pm 1.1$ (stat.) \pm 0.9 (syst. $\left.)\right) \times 10^{-4}$ [3].

\subsection{Search for $h_{b}(1 P)$ in $\Upsilon(3 S) \rightarrow \pi^{+} \pi^{-} X$ decays}

The $h_{b}(1 P)$ has been also searched for in the inclusive dipion decays $\Upsilon(3 S) \rightarrow \pi^{+} \pi^{-} X$. The signal extraction is performed using the recoil mass against the dipion system, defined as: $m_{R}=\sqrt{\left(\sqrt{s}-E^{*}\left(\pi^{+} \pi^{-}\right)\right)^{2}-p^{*}\left(\pi^{+} \pi^{-}\right)^{2}}$, where $E^{*}\left(\pi^{+} \pi^{-}\right)$and $p^{*}\left(\pi^{+} \pi^{-}\right)$are the energy and the 

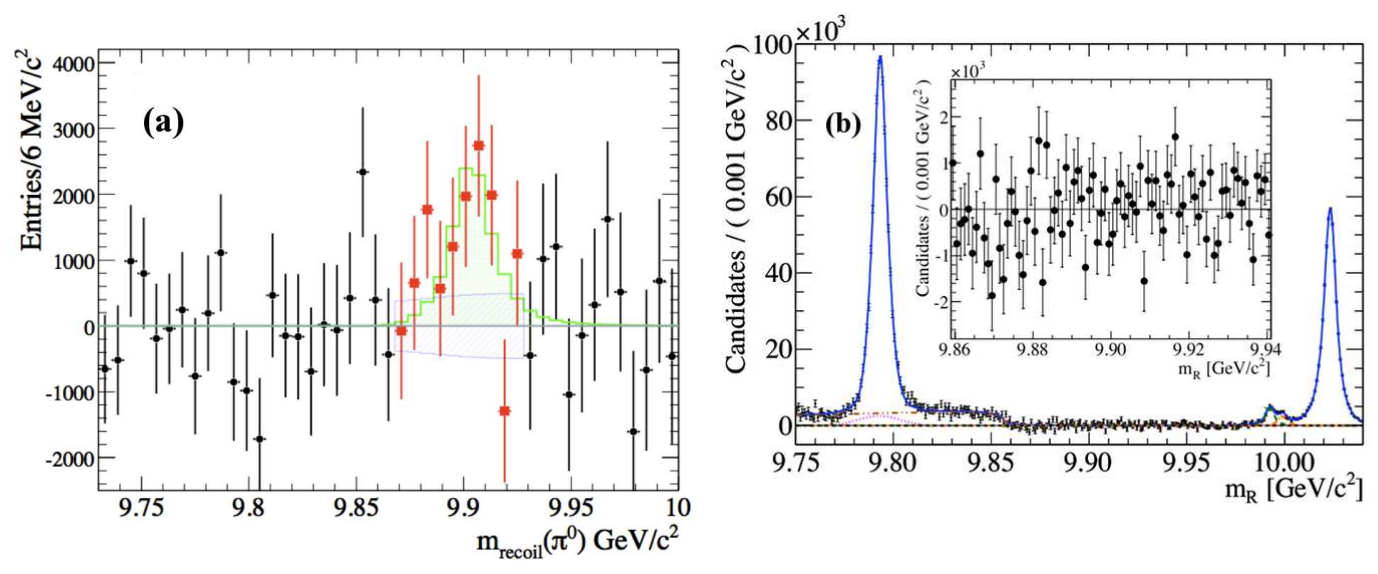

Figure 1: (a) The $m_{\text {recoil }}\left(\pi^{0}\right)$ distribution for data (points, and squares in the signal region), after subtracting background; the shaded histogram is the signal function resulting from the fit to the data, and the area with diagonal shading represents the uncertainties from the background fit. (b) The $m_{R}$ spectrum for data (points) after subtracting the continuum background; the curves represent the $\Upsilon(2 S)_{I S R} \rightarrow \pi^{+} \pi^{-} \Upsilon(1 S)$ (dotted), $K_{S}^{0}$ (double-dot-dashed), $\chi_{b 1}(2 P) \rightarrow \pi^{+} \pi^{-} \chi_{b 1}(1 P)$ (dashed), $\chi_{b 2}(2 P) \rightarrow \pi^{+} \pi^{-} \chi_{b 2}(1 P)$ (dot-dashed), and $\Upsilon(3 S) \rightarrow \pi^{+} \pi^{-} \Upsilon(2 S)$ (long-dashed) components. In the inset, an expanded view of the $h_{b}(1 P)$ region, after subtracting peaking backgrounds.

momentum of the dipion system, calculated in the CM frame. The $h_{b}(1 P)$ signal is expected as a peak on top of a smooth non-peaking background, consisting of continuum events and $K_{S}^{0} \rightarrow \pi^{+} \pi^{-}$ decays, plus several bottomonium dipion transitions: $\Upsilon(3 S) \rightarrow \pi^{+} \pi^{-} \Upsilon(2 S) ; \Upsilon(2 S) \rightarrow \pi^{+} \pi^{-} \Upsilon(1 S)$, with the $\Upsilon(2 S)$ produced either via initial state radiation or through a decay $\Upsilon(3 S) \rightarrow \Upsilon(2 S) X$; and $\chi_{b J}(2 P) \rightarrow \pi^{+} \pi^{-} \chi_{b J}(1 P)$, with $J=1,2$. The distribution of $m_{R}$ is shown in Fig. 1 (b) after subtracting the continuum background, and in the inset after subtracting the peaking bottomonium transitions also. No evidence for the $h_{b}(1 P)$ state is found, and we calculate the $90 \%$ confidence level (CL) upper limit (UL) on the branching fraction, $\mathscr{B}\left(\Upsilon(3 S) \rightarrow \pi^{+} \pi^{-} h_{b}(1 P)\right)<1.2 \times 10^{-4}$ [4]. This result improves by a factor $\sim 10$ the previous best UL, and effectively rules out the prediction for this decay rate [8].

Moreover, this analysis [4] achieves precision measurements of the mass difference between the $\Upsilon(3 S)$ and the $\Upsilon(2 S)$ resonances, and of the branching fractions of several bottomonium dipion transitions. In particular, the transitions $\chi_{b J}(2 P) \rightarrow \pi^{+} \pi^{-} \chi_{b J}(1 P)$, with $J=1,2$, are resolved for the first time. The measured product branching fraction values are $\mathscr{B}\left(\Upsilon(3 S) \rightarrow X \chi_{b 1}(2 P)\right) \times$ $\mathscr{B}\left(\chi_{b 1}(2 P) \rightarrow \pi^{+} \pi^{-} \chi_{b 1}(1 P)\right)=(1.16 \pm 0.07$ (stat. $) \pm 0.12$ (syst. $\left.)\right) \times 10^{-3}$ and $\mathscr{B}\left(\Upsilon(3 S) \rightarrow X \chi_{b 2}(2 P)\right)$ $\times \mathscr{B}\left(\chi_{b 2}(2 P) \rightarrow \pi^{+} \pi^{-} \chi_{b 2}(1 P)\right)=(0.64 \pm 0.05$ (stat. $) \pm 0.08$ (syst. $\left.)\right) \times 10^{-3}$.

\section{Radiative bottomonium transitions using converted photons}

Radiative transitions between bottomonia are well described by effective potential models, in their non-relativistic limit. Nevertheless, there are cases of suppressed electric (i.e. $\Upsilon(3 S) \rightarrow$ $\left.\gamma \chi_{b J}(1 P)\right)$ and of "hindered" magnetic (i.e. $\Upsilon(3 S, 2 S) \rightarrow \gamma \eta_{b}(1 S)$ ) dipole transitions which escape this explanation. The experimental issue in measuring such radiative transitions is the broadening of the photon energies, due to Doppler effect and to detector resolution. The energy resolution 
Table 1: Measured branching fractions for the transitions examined in [5]. The ULs are at the 90\% CL. The first error is statistical, the second systematic, and the third - when present - comes from the uncertainties on secondary branching fractions.

\begin{tabular}{l|c} 
Transition & $\mathscr{B}(\%)[5]$ \\
\hline$\chi_{b 0}(2 P) \rightarrow \gamma \Upsilon(2 S)$ & $<2.9$ \\
$\chi_{b 1}(2 P) \rightarrow \gamma \Upsilon(2 S)$ & $19.5 \pm 1.1_{-1.0}^{+1.1} \pm 1.9$ \\
$\chi_{b 2}(2 P) \rightarrow \gamma \Upsilon(2 S)$ & $8.6_{-0.8}^{+0.9} \pm 0.5 \pm 1.1$ \\
\hline$\chi_{b 0}(2 P) \rightarrow \gamma \Upsilon(1 S)$ & $<1.2$ \\
$\chi_{b 1}(2 P) \rightarrow \gamma \Upsilon(1 S)$ & $9.9 \pm 0.3 \pm 0.4 \pm 0.9$ \\
$\chi_{b 2}(2 P) \rightarrow \gamma \Upsilon(1 S)$ & $7.1 \pm 0.2 \pm 0.3 \pm 0.9$ \\
\hline$\chi_{b 0}(1 P) \rightarrow \gamma \Upsilon(1 S)$ & $<4.6$ \\
$\chi_{b 1}(1 P) \rightarrow \gamma \Upsilon(1 S)$ & $36.2 \pm 0.8 \pm 1.7 \pm 2.1$ \\
$\chi_{b 2}(1 P) \rightarrow \gamma \Upsilon(1 S)$ & $20.2 \pm 0.7_{-0.4}^{+1.0} \pm 1.0$ \\
\hline$\Upsilon(3 S) \rightarrow \gamma \chi_{b 0}(1 P)$ & $2.7 \pm 0.4 \pm 0.2$ \\
$\Upsilon(3 S) \rightarrow \gamma \chi_{b 2}(1 P)$ & $10.6 \pm 0.3 \pm 0.6$ \\
\hline$\Upsilon(3 S) \rightarrow \gamma \eta_{b}(1 S)$ & $0.059 \pm 0.016_{-0.016}^{+0.014}$ \\
$\Upsilon(2 S) \rightarrow \gamma \eta_{b}(1 S)$ & $<0.22$
\end{tabular}

can be significantly improved by using converted photons, that is photons which converted into an $e^{+} e^{-}$pair in the detector material. The photon detection efficiency is $\leq 2.5 \%$. We use the samples of $\Upsilon(3 S)$ and $\Upsilon(2 S)$ resonances, and fit the photon energy spectrum, calculated in the CM frame, in four different regions of interest: for the $\Upsilon(3 S)$ data, between 180 and $300 \mathrm{MeV}$, between 300 and $600 \mathrm{MeV}$, and between 600 and $1100 \mathrm{MeV}$; for the $\Upsilon(2 S)$ data, between 300 and $800 \mathrm{MeV}$. The transitions $\chi_{b J}(2 P) \rightarrow \gamma \Upsilon(2 S, 1 S)$ and $\chi_{b J}(1 P) \rightarrow \gamma \Upsilon(1 S)$, with $J=0,1,2$, are measured with high precision. Moreover, we observe $\Upsilon(3 S) \rightarrow \gamma \chi_{b 0,2}(1 P)$ decays, and search for $\Upsilon(3 S, 2 S) \rightarrow \gamma \eta_{b}(1 S)$. No evidence is found for $\Upsilon(3 S) \rightarrow \gamma \eta_{b}(2 S)$ decays. All the relevant results are summarized in Table 1.

\section{References}

[1] B. Aubert et al. (BABAR Collaboration), Nucl. Instrum. Methods Phys. Res. A 479 (2002) 1.

[2] W. Menges, IEEE Nucl. Sci. Symp. Conf. Rec. 5 (2006) 1470.

[3] J. P. Lees et al. (BABAR Collaboration), arXiv:1102.4565v3 [hep-ex].

[4] J. P. Lees et al. (BABAR Collaboration), Phys. Rev. D84 (2011) 011104(R).

[5] J. P. Lees et al. (BABAR Collaboration), arXiv:1104.5254 [hep-ex].

[6] B. Aubert et al. (BABAR Collaboration), Phys. Rev. Lett. 101 (2008) 071801.

[7] M. B. Voloshin, Sov. J. Nucl. Phys. 43 (1986) 1011.

[8] Y. -P. Kuang, S. F. Tuan, T. -M. Yan, Phys. Rev. D37 (1988) 1210.

[9] S. Godfrey, J. L. Rosner, Phys. Rev. D66 (2002) 014012. 